\title{
Comparación del ruido electroquímico con las técnicas de impedancia y resistencia de polarización en el sistema acero/hormigón ${ }^{(\bullet)}$
}

\author{
A. Bautista ${ }^{(*)}$, A. Vergara ${ }^{(* *)}$, J.V. Dávila ${ }^{(* *)}$, L. Mariaca $^{(* * *)}$ y J.A. González ${ }^{(*)}$
}

Resumen En este trabajo se estudia mediante diferentes técnicas electroquímicas la velocidad de corrosión de armaduras de acero activas y pasivas embebidas en hormigón con diferentes grados de humedad. Se pone énfasis en la validez del ruido electroquímico como método para cuantificar la intensidad del ataque y en las ventajas que esta técnica ofrece. Además, se presentan datos que demuestran la proporcionalidad existente, en el sistema estudiado, entre la velocidad de corrosión y el ruido en corriente, y la independencia entre este parámetro cinético y el ruido en potencial.

Palabras clave: Ruido electroquímico. Técnicas electroquímicas. Hormigón armado. Velocidad de corrosión.

\section{Comparison of electrochemical noise with impedance and polarization resistance techniques in the steel/concrete system}

\begin{abstract}
This paper studies the corrosion rates of active and passive rebars embedded in concrete in various degrees of wetness using different electrochemical techniques. The ability of the electrochemical noise to quantificate the intensity of the attack and the advantages of this method are stressed. Data that prove that the corrosion rate is proportional to the current noise and independent to the potential noise in this system are also shown.
\end{abstract}

Keywords: Electrochemical noise. Electrochemical techniques. Reinforced concrete. Corrosion rate.

\section{INTRODUCCIÓN}

Tradicionalmente, la investigación de la corrosión en las estructuras de hormigón armado se realizaba utilizando procedimientos visuales o gravimétricos. Estos métodos tienen la desventaja de requerir largos tiempos de exposición para poder evaluar la velocidad de corrosión y de que, al ser destructivos, sólo permiten obtener un único dato a partir de cada probeta. Las técnicas electroquímicas, que han logrado un gran desarrollo en las últi-

(•) Trabajo recibido el día 4 de septiembre de 1996

(*) Centro Nacional de Investigaciones Metalúrgicas (CSIC). Avda. de Gregorio del Amo, 8. 28040-Madrid (España).

(**) Universidad Nacional de Ingeniería. Lima (Perú).

(***) Centro de Investigaciones Eléctricas. Cuernavaca (México). mas décadas, permiten evaluar la velocidad de corrosión de las armaduras antes de que aparezcan los síntomas visibles del ataque y, al ser no destructivas, seguir la evolución del proceso a lo largo del tiempo.

Los métodos electroquímicos empleados en el estudio de la corrosión del acero embebido en hormigón van desde la simple medida de los potenciales de electrodo -que puede suministrar indicaciones sobre el estado activo o pasivo, pero no una información cuantitativa acerca de la velocidad de corrosión- hasta las técnicas de corriente continua (curvas de polarización y escalones de potencial) y de corriente alterna (espectroscopía de impedancia electroquímica, EIE) que miden la velocidad instantánea de corrosión.

Desde que en 1959 Kaesche (1) y Baümel y Engell (2) realizaron por primera vez curvas de polarización en probetas de hormigón, este método 
ha sido empleado por muchos investigadores. Las elevadas polarizaciones, de centenares de milivoltios, necesarias para trazar estas curvas, pueden modificar, sin embargo, la superficie de la armadura.

La medida de la resistencia de polarización, $R_{\mathrm{p}}$, a partir de la aplicación de escalones de potencial, según el método propuesto por Stern y Geary en 1957 (3), no se empezó a emplear en el sistema acero-hormigón hasta los años 70 (4). Esta técnica permite obtener información sobre la velocidad de corrosión en tan solo unos pocos segundos, pero también perturba al electrodo, aunque en mucha menor medida que las curvas de polarización (se suelen aplicar pulsos de $10 \mathrm{mV}$ ) (5).

La EIE se introdujo en el estudio de la corrosión de las armaduras de hormigón en el decenio de los 80 (6 y 7). Este método, aunque suele ofrecer una información bastante completa del proceso de corrosión, implica el uso de equipos caros y la aplicación de perturbaciones sinusoidales de pequeña amplitud (5-10 mV, normalmente).

La necesidad de polarizar al electrodo para obtener información sobre el proceso de corrosión, con los riesgos de alterar el desarrollo natural del fenómeno que eso conlleva, afecta a la fiabilidad de los resultados obtenidos mediante técnicas de corriente continua y alterna. El ruido electroquímico, sin embargo, ofrece la posibilidad de obtener información sin necesidad de desplazar al electrodo de su potencial de corrosión ni de introducir ninguna señal ajena al sistema. Esta técnica se limita a registrar las oscilaciones espontáneas del potencial y/o de la corriente. Las oscilaciones se producen porque en la superficie del electrodo que se corroe tienen lugar cambios localizados, que originan breves alteraciones de la resistencia interfacial (8). Estas variaciones del valor de la resistencia en cada zona pequeña del electrodo dan lugar a fluctuaciones de la corriente de las semirreacciones anódica y catódica en esa zona. Entonces la intensidad anódica y la catódica dejan de ser iguales en valor absoluto y se produce una desviación instantánea y localizada de la intensidad de corriente global cero. La densidad de corriente a circuito abierto del electrodo viene dada por la suma de las densidades de todos los procesos anódicos y catódicos que tienen lugar sobre su superficie, y como las intensidades de estos procesos presentan pequeñas fluctuaciones, el registro de la densidad de corriente presenta un cierto "ruido". Además, estas desviaciones de la intensidad de corriente hacen que el potencial del electrodo se desplace hacia valores ligeramente más positivos o más negativos, de tal forma que se compense la diferencia entre las intensidades de las semirreacciones anódica y catódica. Esto hace que el valor del potencial oscile, es decir, da lugar al ruido en potencial.
A partir de las fluctuaciones aleatorias de los parámetros electroquímicos de un sistema, se ha tratado de obtener información sobre el mecanismo de corrosión (8-14) y sobre la intensidad del ataque (9, 10 y 15-21). Los métodos propuestos para interpretar los datos de ruido electroquímico son variados, y las conclusiones obtenidas por diferentes investigadores al relacionar el ruido electroquímico tanto con el mecanismo de corrosión (10 y 11) como con la velocidad del proceso (11 y 18) han resultado ser, a veces, aparentemente contradictorias. En este trabajo se comparan las velocidades de corrosión obtenidas para el sistema acero/hormigón mediante la medida de resistencia de la polarización y EIE con las obtenidas a partir del ruido electroquímico, con el fin de comprobar la fiabilidad de este último método. La obtención de velocidades de corrosión a partir de datos de ruido electroquímico se ha realizado utilizando la llamada resistencia de polarización de ruido, $R_{\mathrm{r}}$, cuyo valor viene dado por la siguiente expresión:

$$
R_{\mathrm{r}}=\sigma_{\mathrm{v}} / \sigma_{\mathrm{i}}
$$

donde: $\sigma_{\mathrm{v}}$ es la desviación estándar del ruido en potencial y $\sigma_{\mathrm{i}}$ es la desviación estándar del ruido en corriente. La $R_{\mathrm{r}}$ parece coincidir con la resistencia de polarización del metal en algunos sistemas (1719), y en otros simplemente muestra una cierta proporcionalidad con este parámetro (20 y 21$)$.

\section{DESARROLLO EXPERIMENTAL}

\subsection{Materiales}

Los ensayos se realizaron en probetas de mortero de $2 \times 5,5 \times 8 \mathrm{~cm}$, fabricadas con cemento Portland-450, con relaciones cemento/arena de $1 / 3$ y agua/cemento de 0,5. En cada probeta se habían embebido dos armaduras idénticas de acero al carbono y un alambre de acero inoxidable (Fig. 1). A una parte de las probetas se le añadió durante el amasado un $2 \%$ en peso de iones $\mathrm{NO}_{2}^{-}$como $\mathrm{NaNO}_{2}$. Posteriormente, todas las probetas se sumergieron en agua de mar. Los cloruros absorbidos en la red de poros del mortero provocaron la corrosión en estado activo del acero de parte de las probetas, aquellas a las que no se les había añadido $\mathrm{NO}_{2}^{-}$, mientras que las amasadas con estos iones, que tienen un fuerte efecto inhibidor de la corrosión (22), mantuvieron la pasividad de las armaduras.

Las probetas continuaron sumergidas en agua de Madrid durante unos días y luego se dejaron secar en la atmósfera de laboratorio, con el fin de estudiar la capacidad de los diferentes métodos para detectar las variaciones de la velocidad de corrosión del acero que tienen lugar al variar la humedad del mortero (23). 

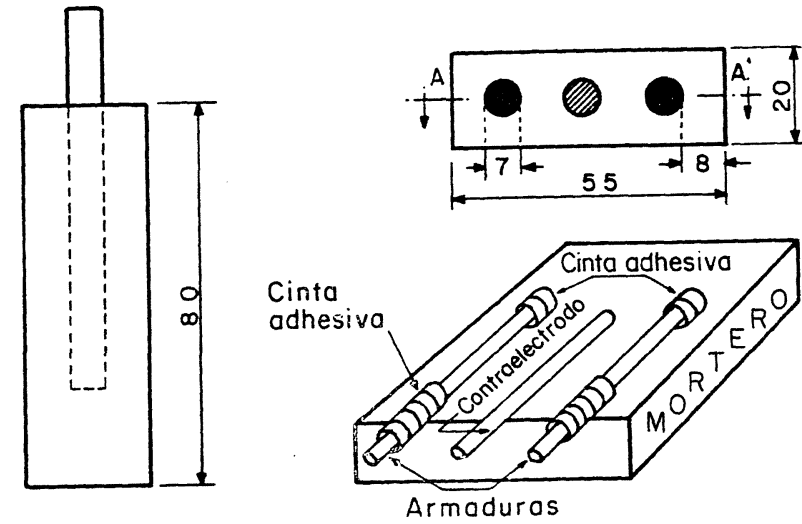

FIG. 1.- Esquema del tipo de probetas usadas en la investigación.

FIG. 1.- Schematic depiction of the specimens studied.

\subsection{Métodos}

Las curvas de polarización se obtuvieron con un potenciostato 1286 , con una velocidad de barrido de $1 \mathrm{mV} / \mathrm{s}$. La caída óhmica que tiene lugar debida a la resistividad del mortero, se compensó automáticamente con el "positive feed back" del potenciostato.

Las medidas de resistencia de polarización se llevaron a cabo aplicando escalones de potencial de $10 \mathrm{mV}$ y midiendo la variación de la corriente a los 15 s de haber aplicado el pulso. Para ello, se utilizó el mismo potenciostato empleado en las curvas de polarización y se compensó la caída óhmica con el mismo método.

Los diagramas de impedancia se obtuvieron con un potenciostato PAR 273A conectado a un analizador de respuesta a la frecuencia SI 1255, realizando barridos desde $100 \mathrm{kHz}$ hasta $1 \mathrm{mHz}$.

Tanto en la obtención de las curvas de polarización como en las medidas de $R_{\mathrm{p}}$ e impedancia, se usó como electrodo de trabajo una de las armaduras de acero al carbono de la probeta, como contraelectrodo el alambre de acero inoxidable y como electrodo de referencia uno de calomelanos saturado situado sobre la superficie exterior del mortero.

Las medidas de ruido electroquímico se realizaron con un microamperímetro de resistencia cero, modelo AutoZRA, de ACM Instruments. Se registraron simultáneamente las oscilaciones del potencial y la corriente en el potencial de corrosión del sistema. Los datos se adquirieron cada $0,7 \mathrm{~s}$, hasta completar ficheros de 1.024 datos para cada parámetro. Antes de realizar el análisis estadístico de los datos se llevó a cabo un proceso de eliminación de la tendencia, con el fin corregir la variación del valor medio de los parámetros electroquímicos del sistema durante el tiempo de adquisición de los datos, y evitar el error que supondría considerar ruido lo que es una evolución no aleatoria del potencial o de la corriente. Para ello, los datos obtenidos se ajustaron a una recta y se consideró como ruido la diferencia en cada instante entre el valor medido y el valor de la recta ajustada. Estos valores se usaron para calcular las desviaciones estándar y se transfirieron al campo de las frecuencias mediante la transformada rápida de Fourier.

Los datos de ruido electroquímico se obtuvieron usando una de las armaduras de la probeta en estudio como electrodo de trabajo y haciendo actuar a la otra armadura de la misma probeta como electrodo de referencia y contraelectrodo a la vez.

\section{RESULTADOS}

Las curvas de polarización de la figura 2 muestran la gran diferencia que existe en los comportamientos de las armaduras embebidas en hormigón con cloruros y las embebidas en hormigón con cloruros y nitritos cuando el mortero está húmedo. Mientras que en las primeras una alteración de unos $100 \mu \mathrm{A}$ en la intensidad de corrosión no logra modificar, de forma detectable, el potencial a circuito abierto del sistema, en las segundas una variación de ese orden en la intensidad de corrosión es suficiente para desplazar en centenares de milivoltios su potencial, es decir, las armaduras resultan mucho más polarizables si su velocidad de corrosión es baja.

Además, la recuperación después de una perturbación tambiên es muy distinta en armaduras activas y pasivas cuando la humedad del mortero es elevada. Comparando las figuras 3 y 4, se aprecia que, tras la aplicación de un escalón de potencial de

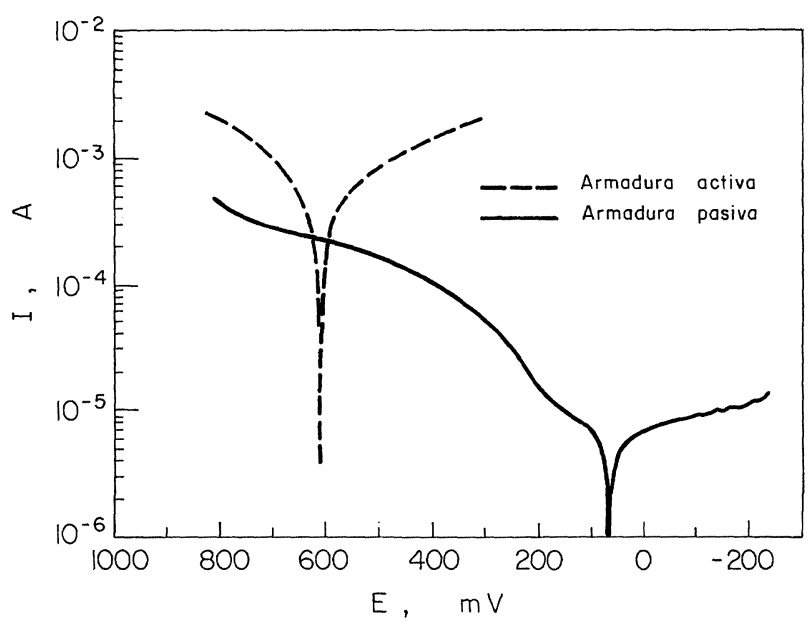

FIG. 2.- Curvas de polarización correspondientes a armaduras activas y pasivas cuando el grado de humedad del hormigón es alto.

FIG. 2.- Polarization curves for active and passive rebars in wet concrete. 


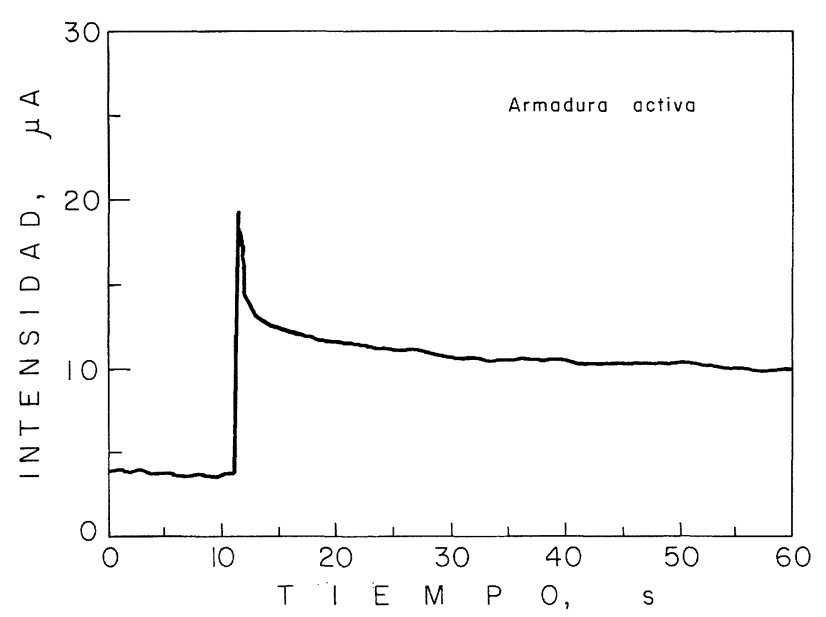

FIG. 3.- Evolución de la intensidad de corriente tras un pulso de potencial en armaduras activas cuando el grado de humedad del hormigón es alto.

FIG. 3.- Evolution of the current intensity after a potential pulse for active rebars in wet concrete.

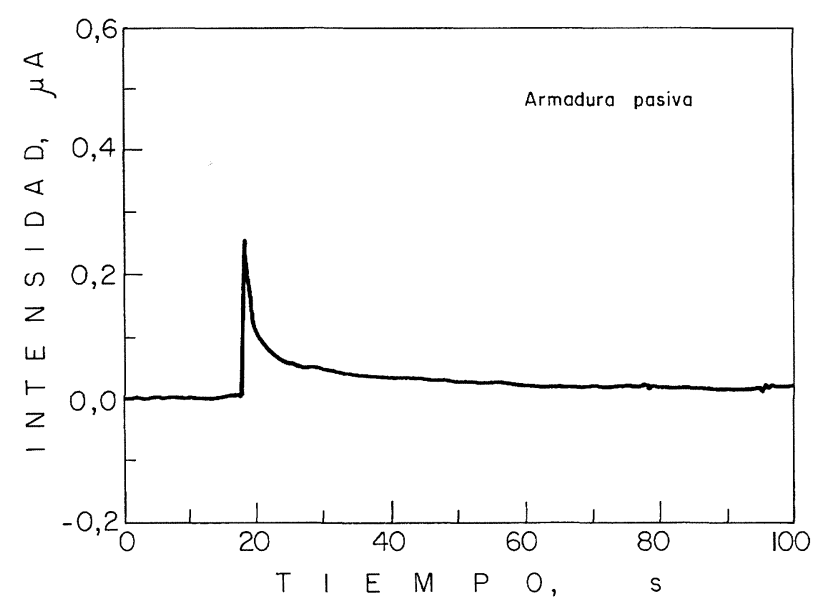

FIG. 4.- Evolución de la intensidad de corriente tras un pulso de potencial en armaduras pasivas cuando el grado de humedad del hormigón es alto.

FIG. 4.- Evolution of the current intensity after a potential pulse for passive rebars in wet concrete.

$10 \mathrm{mV}$ a las armaduras activas, la intensidad de corriente se estabiliza en unos pocos segundos, mientras que, tras ese mismo pulso de potencial, la intensidad de corriente correspondiente a las armaduras pasivas tarda mucho en estabilizarse. Esto hace que la determinación del valor de $R_{\mathrm{p}}$, y por tanto de la velocidad de corrosión, no ofrezcan dudas cuando las armaduras se corroen activamente, pero que presenten una gran indeterminación cuando la velocidad de corrosión es muy pequeña, porque en ese caso el valor de $R_{\mathrm{p}}$ calculado depende sensiblemente del tiempo transcurrido entre la aplicación del escalón de potencial y la lectura de la variación de la intensidad de corriente. Además, la variación de la intensidad de corriente que se produce en el caso de las armaduras pasivas está próxima al límite de sensibilidad del aparato.

Mediante EIE se obtienen diagramas como el de la figura 5 cuando las armaduras se corroen activamente. En ellos se insinúa un primer semicírculo, a frecuencias altas, que corresponde a la resistividad del mortero, y otro, claramente definido a frecuencias más bajas, que corresponde al proceso de corrosión de las armaduras. Por el contrario, en los diagramas de impedancia de armaduras con una baja velocidad de corrosión (Fig. 6), el semicírculo correspondiente al proceso electroquímico, de diámetro mucho mayor que en el caso anterior, apenas se insinúa. En consecuencia, cuando las armaduras se corroen activamente se puede obtener sin problemas la resistencia a la transferencia de carga, $R_{\mathrm{t}}$, y por tanto la velocidad de corrosión, a partir del diámetro del semicírculo de bajas frecuencias que aparece en su espectro de impedancias. Cuando la armadura está pasiva o el grado de humedad del mortero es muy bajo, la mala definición del semicírculo de bajas frecuencias hace imposible estimar, siquiera aproximadamente, la $R_{\mathrm{t}}$ y la velocidad del proceso.

Los resultados obtenidos mediante ruido electroquímico revelan una clara diferencia entre el ruido en corriente de las armaduras activas y pasivas cuando el grado de humedad del mortero es alto. Esta diferencia se puede apreciar sencillamente comparando los resultados del análisis estadístico o las representaciones gráficas de densidad espectral

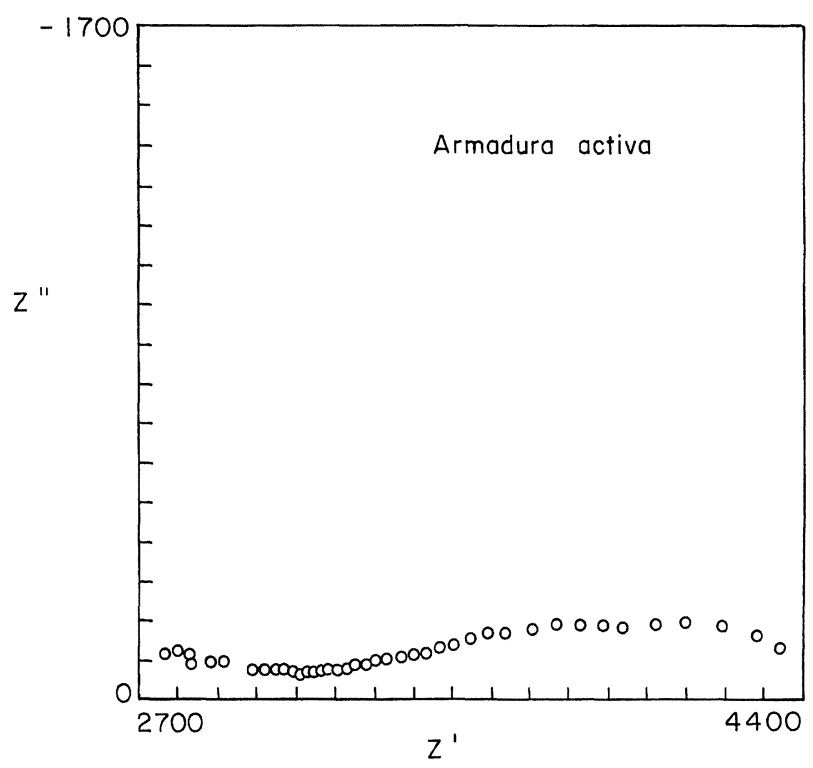

FIG. 5.- Diagramas de impedancia correspondientes a armaduras activas cuando el grado de humedad del hormigón es alto.

FIG. 5.- Impedance diagrams for active rebars in wet concrete. 


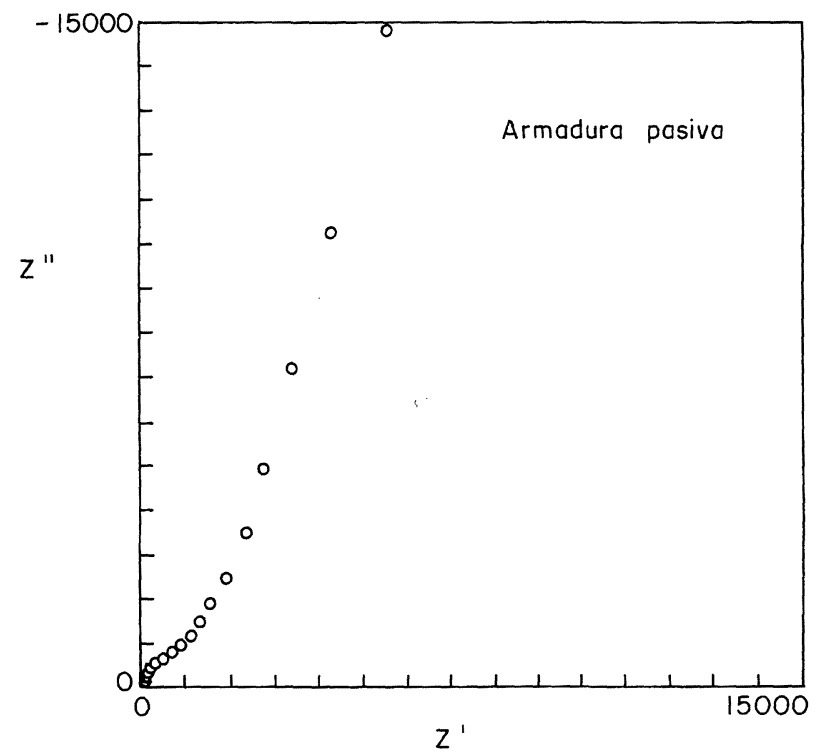

FIG. 6.- Diagramas de impedancia correspondientes a armaduras pasivas cuando el grado de humedad del hormigón es alto.

FIG. 6.-Impedance diagrams for passive rebars in wet concrete.

de potencia obtenidas en ambos casos (Fig. 7a). El ruido en corriente, que puede cuantificarse a través de $\sigma_{\mathrm{i}}$ y está relacionado con el área bajo las curvas de densidad espectral de potencia, aumenta cuando lo hace la velocidad de corrosión del sistema acero/hormigón. El ruido en potencial, sin embargo, mantiene constante su desviación estándar y sus representaciones gráficas de densidad espectral de potencia no se modifican significativamente (Fig. $7 b$ ), cualquiera que sea la velocidad de corrosión de las probetas.

La comparación de los valores de $R_{\mathrm{r}}$ calculados aplicando la expresión [1] muestran buena correlación con los valores de $R_{\mathrm{p}}$ obtenidos mediante pulsos de potencial y con los de $R_{\mathrm{t}}$ deducidos a partir de diagramas de EIE, tanto para armaduras pasivas como para activas, dentro del amplio intervalo de velocidades de corrosión que se ha logrado reproducir variando la humedad del mortero. Como se puede ver en la figura 8 , los resultados obtenidos se disponen en torno a la línea de trazos que representa la relación ideal $1 / 1$ entre los valores de $R_{\mathrm{r}}$ y los de $R_{\mathrm{p}}$ o $R_{\mathrm{t}}$.

La variación de $R_{\mathrm{r}}$ con la velocidad de corrosión parece venir dada exclusivamente en el sistema acero/hormigón por la disminución de $\sigma_{i}$ al aumentar la resistencia a la polarización del acero, mientras que $\sigma_{\mathrm{v}}$ permanece constante (en el caso de las probetas ensayadas siempre en torno a $2 \cdot 10^{-2} \mathrm{mV}$ ) cualquiera que sea la resistencia a la polarización de las armaduras (Fig. 9).
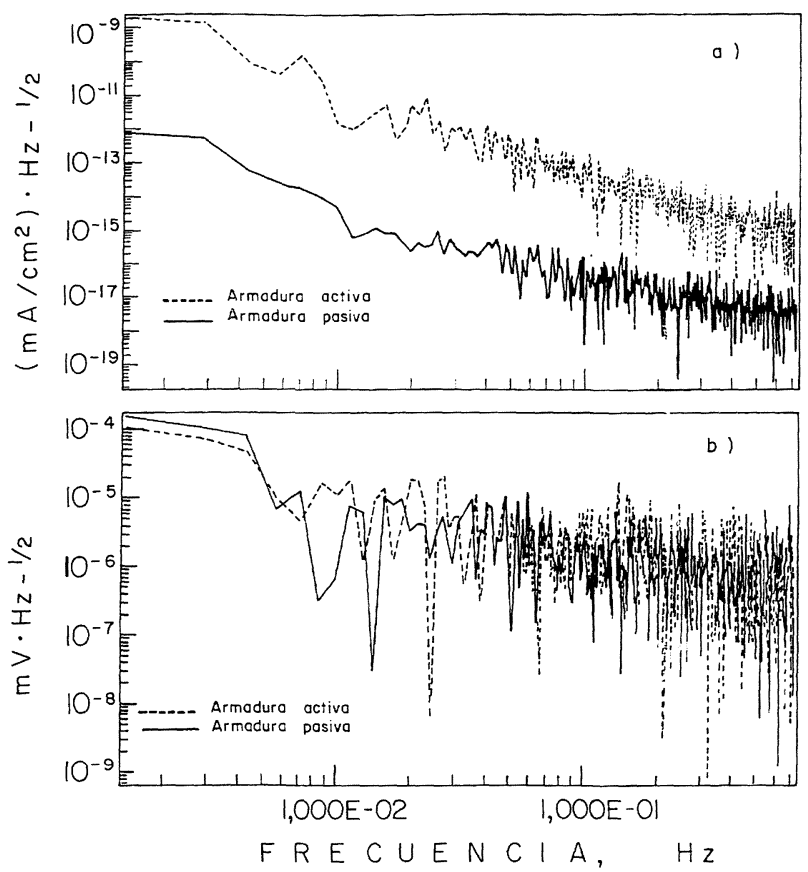

FIG. 7.- Densidad espectral de potencia del ruido: a) en corriente, y b) en potencial, de armaduras activas y pasivas cuando el grado de humedad del hormigón es alto.

FIG. 7.- Power spectral density: a) the current noise, and b) the potential, noise of active and passive rebars in wet concrete.

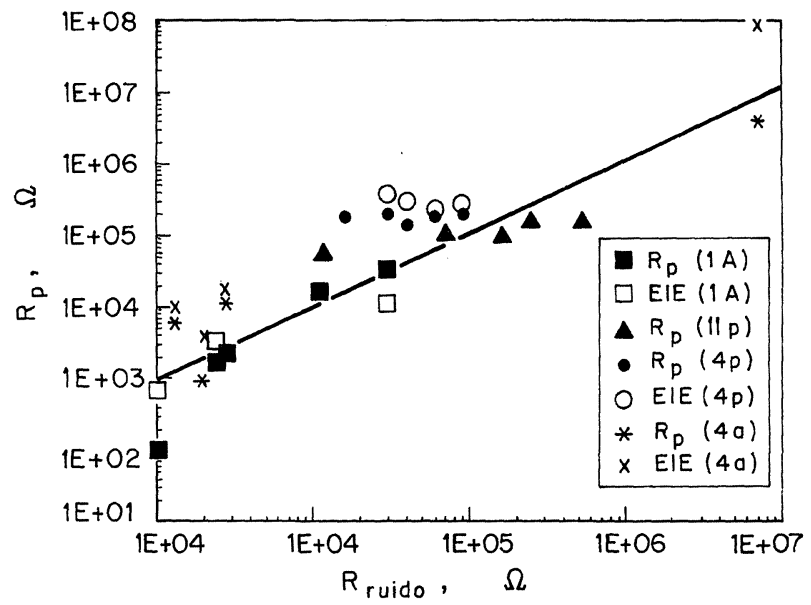

FIG. 8.- Comparación entre los valores de resistencia de polarización obtenidos mediante ruido electroquímico y los obtenidos mediante impedancia y pulsos de potencial para armaduras activas y pasivas embebidas en hormigón con diferentes grados de humedad.

FIG. 8.- Comparison between the polarization resistance values obtained using electrochemical noise and the ones obtained using impedance and potential pulses for active and passive rebars embedded in concrete in various degrees of wetness. 


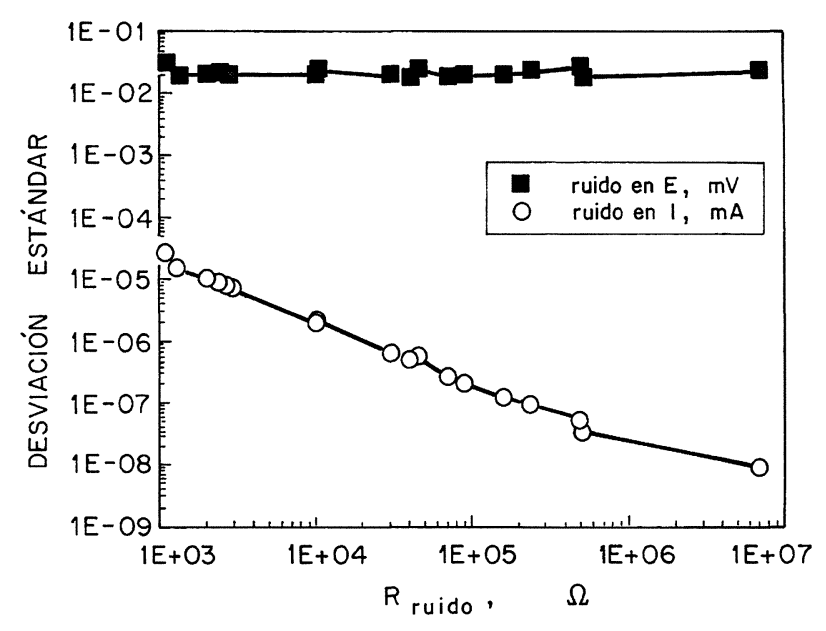

FIG. 9.- Relación de las desviaciones estándar del ruido en potencial y en corriente con la resistencia de polarización de ruido.

FIG. 9.- Relationship between potential and current standard deviations and noise polarization resistance.

\section{DISCUSIÓN}

Las polarizaciones pueden provocar la alteración, más o menos irreversible, de parámetros del sistema tales como el microperfil superficial, la absorción o desorción de sustancias en lugares activos de la superficie, en los procesos redox superpuestos al de corrosión, etc. Estas alteraciones son tanto mayores cuanto mayor es la polarización, y cuando se trata de medir mediante los métodos electroquímicos tradicionales la velocidad de corrosión de un electrodo pasivo, fácilmente polarizable (Fig. 2), el riesgo de modificar al sistema no es despreciable. Esto, junto con el hecho de que los métodos del pulso de potencial y la EIS resultan adecuados para determinar la velocidad de corrosión de sistemas que se corroen activamente (Figs. 3 y 5), pero presentan dificultades a la hora de cuantificar la intensidad del ataque cuando ésta es pequeña (Figs. 4 y 6), justifica el interés despertado por el ruido electroquímico.

La buena correlación encontrada entre los valores de $R_{\mathrm{r}}$ calculados a partir de los datos de ruido electroquímico con los valores de $R_{\mathrm{p}}$ y $R_{\mathrm{t}}$ obtenidos mediante otras técnicas ya muy utilizadas en el estudio de la corrosión del hormigón armado (Fig. 8) demuestra que en este caso $R_{\mathrm{r}}$ no es simplemente proporcional a la resistencia de polarización del metal, sino que puede identificarse directamente con este parámetro y utilizarse para calcular la intensidad de corrosión, $i_{\text {corr }}$, mediante la fórmula propuesta por Stern y Geary (3):

$$
i_{\text {corr }}=B / R_{\mathrm{p}} \approx B / R_{\mathrm{r}}
$$

donde: $B$ es una constante cuyo valor suele oscilar en el caso de las estructuras de hormigón armado en torno a $26 \mathrm{mV}$ cuando el sistema está activo, y en torno a $52 \mathrm{mV}$ cuando está pasivo (5).

La sencillez del cálculo de la $R_{\mathrm{r}}$, que permite cuantificar la velocidad de corrosión incluso en el caso de armaduras pasivas, junto con la asequibilidad económica del equipo necesario para obtener los datos y la fiabilidad que aporta el hecho de que la medida no perturba el sistema, hacen que este método resulte una alternativa ventajosa a las técnicas actualmente implantadas en el estudio de la corrosión del hormigón armado.

Por otro lado, es necesario destacar que, a pesar de que tradicionalmente los estudios de ruido electroquímico se centraron en las oscilaciones de potencial para obtener información del proceso, el ruido en corriente resulta fundamental a la hora de determinar la velocidad de corrosión en el sistema acero/hormigón. Es fácil entender que, en cualquier sistema, cuando la velocidad de corrosión es alta, la mayor actividad de las micropilas de corrosión dará lugar a que las oscilaciones de la intensidad de corriente en torno a su valor medio sean mayores. El comportamiento del ruido en potencial, sin embargo, es mucho más difícil de predecir, pues viene determinado por dos efectos contrapuestos. Por un lado, en los sistemas pasivos, al ser mucho más polarizables que los activos, las oscilaciones de potencial que produce una misma variación de corriente son mucho mayores, pero por otro, las oscilaciones de corriente son mucho más pequeñas cuando la velocidad de corrosión es baja. En concordancia con cuanto antecede, en el caso de las probetas activas estudiadas, el ruido en corriente variaba su amplitud (Fig. 9) y su espectro de frecuencias (Fig. 7a) con la velocidad de corrosión, tal y como era de esperar, mientras que todas las probetas presentaban un ruido en potencial de la misma amplitud (Fig. 9) y la misma densidad espectral de potencia (Fig. 7b), independientemente de la velocidad a la que se estuvieran corroyendo sus armaduras. Es decir, en este caso los efectos del aumento de las oscilaciones de corriente y de la disminución de la polarizabilidad con la velocidad de corrosión se compensaban y el ruido en potencial era siempre similar. Por tanto, la sensibilidad del ruido electroquímico como técnica para distinguir distintas velocidades de corrosión de acero embebido en hormigón viene dada por la proporcionalidad directa entre la amplitud de las oscilaciones de la intensidad de corriente en el potencial de corrosión, medida a través de $\sigma_{i}$, y la intensidad de corrosión. El estudio del ruido en potencial ha servido simplemente, en este caso, para que al multiplicar la inversa de $\sigma_{\mathrm{i}}$ por $\sigma_{\mathrm{v}}$ se pueda obtener un valor de resistencia que coincida con la $R_{\mathrm{p}}$ de las armaduras. 


\section{CONCLUSIONES}

- El ruido electroquímico permite cuantificar la velocidad de corrosión del sistema acero/hormigón, tanto en el caso de armaduras activas como en el de armaduras pasivas. Esta cuantificación se puede hacer de forma rápida y sencilla calculando $R_{\mathrm{r}}$, que en este caso se puede identificar directamente con la $R_{\mathrm{p}}$ del metal.

- El ruido en potencial presenta siempre la misma desviación estándar y el mismo espectro en el campo de las frecuencias en todas las probetas estudiadas, independientemente de la composición del mortero o del grado de humedad de éste.

- El ruido en corriente aumenta cuando lo hace la velocidad de corrosión de las armaduras.

- La disminución del valor de $R_{\mathrm{r}}$ con el aumento de la intensidad del ataque viene dada exclusivamente por la variación de $\sigma_{\mathrm{i}}$ con la velocidad de corrosión.

\section{REFERENCIAS}

(1) KAESCHE, H. Zement-Kalk-Gips, 12 (7), 1959: 298-294.

(2) Baümel, A. y Engell, H.J. Archiv. Eisenhüttenwes., 30 (7), 1959: 417.

(3) Stern, M. y Geary, A.L. J. Electrochem. Soc., 104 (1), 1957: 56-63.

(4) Gouda, W.K., Sharter, M.A. y Mikhail, R.S.H. Cem. Concr. Res., 5 (1), 1975: 1-7.

(5) Gonzalez, J.A. y Fullea, J. Corr. Proct., 5 (5), 1974: 273-289.

(6) Wenger, F., Galland, J., y Lemoine, L. International Symposium on Behavior of Offshore Concrete Structures. Brest (Francia), Oct., 1980.

(7) Wenger, F., Lemoine, L. y Galland, J. $9^{\text {th }}$ International Congress on Metal Corrosion. Vol. 3. Toronto (Canadá), Jun. 1984: 225-228.
(8) Bertocci, U. y Huet, F. Corrosion, 51 (2), 1995: 131144.

(9) Garcia O., E., Uruchurtu, J. y Genesca, J. Rev. Metal., Madrid, 31 (6), 1995: 361-367.

(10) Hladky, K. y Dawson, J.L. Corr. Sci., 22 (3), 1982: 231237.

(11) Legat, A. y Dolecek, V. Corrosion, 51 (4), 1995: 295300.

(12) Cottis, R.A. у Loto, C.A. Corrosion, 46 (1), 1990: 1218.

(13) Uruchurtu, J. Corrosion, 47 (6), 1991: 472-479.

(14) Searson, P.C. y Dawson, J.L. J. Electrochem. Soc., 135 (8), 1988: 1.908-1.915.

(15) Hardon, R.G., Lambert. P. y Page, C.L. Br. Corr. J., 23 (4), 1988: 225-228.

(16) Metikos-Hukovic, M., LonCar. M. y Zevnik, C. Werks. Korr., 40 (8), 1989: 494-499.

(17) Mansfeld, F. y XiaO, H. J. Electrochem. Soc., 140 (8), 1993: 2.205-2.209.

(18) Bautista, A., Mariaca, L., Rodriguez, P. y Gonzalez, J.A. $5^{\circ}$ 'Congreso Iberoamericano de Corrosión y Protección. Tenerife (España), Oct. 1995.

(19) Eden, D.A. y Rothwell, A.N. Electrochemical Noise Data: Analysis, Interpretation and Presentation. Paper No. 292, CORROSION/92. NACE. Houston, TX (EE.UU.), 1992.

(20) Xiao, H. y Mansfeld, F. J. Electrochem. Soc., 141 (9), 1994: 2.332-2.337.

(21) Lumsdem, J.B., Kending, M.W. y Jeanjaquet, S. Electrochemical Noise for Carbon Steel in Sodium Chloride Solutions-Effect of Chloride and Oxigen Activity. Paper No. 224. CORROSION/92. NACE. Houston, TX (EE.UU.), 1992.

(22) Ramirez, E., Otero, E. y Gonzalez, J.A. Br. Corr. J., 28 (2), 1993: 121-124.

(23) Gonzalez, J.A., LoPez, W. y Rodriguez, P. Corrosion, 49 (12), 1993: 1.004-1.010. 